\title{
Mouvements oscillatoires de corps en ascension dans un fluide peu visqueux : l'effet du rapport de forme
}

\author{
Pedro C. Fernandes ${ }^{a}$, Patricia Ern, Frédéric Risso et Jacques Magnaudet \\ Institut de Mécanique des Fluides de Toulouse, UMR 5502 CNRS-INP-UPS, Allée du Prof. Camille Soula, \\ 31400 Toulouse, France
}

Reçu le 25 février 2005, accepté le 21 mars 2005

\begin{abstract}
Résumé - On observe souvent dans la nature que l'ascension ou la chute d'un corps peut présenter des mouvements oscillatoires (spirale, zigzag) ou plus désordonnés. Nous nous sommes penchés sur les causes des instabilités du mouvement d'un corps en ascension sous l'effet de la gravité, dans un fluide au repos. Nous avons conduit une étude expérimentale des mouvements oscillatoires de corps légers montant librement dans un fluide peu visqueux. Des résultats originaux concernant la cinématique de cylindres minces sont présentés ici pour une large gamme de nombres d'Archimède (flottabilité sur effets visqueux) et du rapport de forme (diamètre sur épaisseur). Nous avons analysé les oscillations de la vitesse et de l'orientation des cylindres (fréquences, amplitudes et différences de phases), ce qui a mis en évidence l'effet crucial du rapport de forme dans le couplage entre la translation et la rotation.
\end{abstract}

Mots clés : Corps mobile / oscillations auto-entretenues / instabilité de trajectoire / sillage

\begin{abstract}
Oscillatory motions of freely-moving bodies rising in a low-viscous fluid: the role of the aspect ratio. In many situations, freely falling or rising particles exhibit oscillatory motions: spiral, zigzag or tumbling. We are concerned with the causes of these path instabilities for particles moving under the effect of buoyancy in a fluid otherwise at rest. The oscillatory motion of light particles rising freely in a slightly viscous fluid was investigated experimentally. Original results concerning the kinematics of flat cylinders are reported for a wide range of the Archimedes number (buoyancy vs. viscous effects) and aspect ratio (diameter-to-height ratio). In this paper, we focus on the particle velocity and orientation oscillations (frequencies, amplitudes and phase differences), underlining the crucial effect of the body aspect ratio on the complex coupling between translation and rotation.
\end{abstract}

Key words: Freely-moving body / self-sustained oscillations / trajectory instability / wake

\section{Introduction}

Les trajectoires en spirale ou zigzag de corps en mouvement dans un fluide sont souvent observées dans la nature : l'ascension de bulles dans l'eau ou la chute de feuilles dans l'air en sont des exemples classiques. Ces mouvements de chute ou d'ascension non rectilignes ont attiré, au cours de l'histoire, l'attention de grands savants comme Léonard de Vinci [1], Newton [2] et Maxwell [3]. Mais, ce n'est que maintenant que les moyens de mesure sophistiqués permettent une description précise de ces mouvements.

Dans ce travail, nous avons étudié expérimentalement le processus de déstabilisation du mouvement d'un corps

\footnotetext{
a Auteur correspondant : cordeiro@imft.fr
}

montant dans un fluide au repos en utilisant une technique de trajectographie 3D. Le mouvement d'un corps mobile se déplaçant sous l'effet de la gravité dépend de trois paramètres : le nombre d'Archimède, le rapport de forme et le rapport des densités du solide et du fluide. Si l'on considère par exemple le cas d'une sphère libre $[4,5]$, on sait qu'au-delà d'un certain nombre d'Archimède son sillage devient instable et les forces de traînée et de portance instationnaires peuvent engendrer les mouvements oscillatoires observés. Ces oscillations sont d'autant plus marquées que le corps est léger c'est-à-dire quand le rapport des densités du solide et du fluide $\left(\rho_{\mathrm{s}} / \rho_{\mathrm{f}}\right)$ est petit, comme dans le cas d'une bulle $[6,7]$. De plus, pour un corps qui n'est pas sphérique, on sait que l'anisotropie du tenseur d'inertie ajoutée peut aussi créer des oscillations auto-entretenues en couplant les équations de translation 


\section{Nomenclature}

\begin{tabular}{|ll|}
\hline$d$ & diamètre du corps $(\mathrm{m})$ \\
$h$ & hauteur du corps $(\mathrm{m})$ \\
$d / h$ & rapport de forme du corps \\
$r_{\mathrm{eq}}$ & rayon équivalent du corps $(\mathrm{m})$ \\
$\rho_{\mathrm{s}}$ & densité du corps solide $\left(\mathrm{kg} \cdot \mathrm{m}^{-3}\right)$ \\
$\rho_{\mathrm{f}}$ & densité du fluide $\left(\mathrm{kg} \cdot \mathrm{m}^{-3}\right)$ \\
$\Delta \rho$ & différence des densités du fluide et du corps solide $\left(\mathrm{kg} \cdot \mathrm{m}^{-3}\right)$ \\
$\nu_{\mathrm{f}}$ & viscosité cinématique du fluide $\left(\mathrm{m}^{2} \cdot \mathrm{s}^{-1}\right)$ \\
$g$ & accélération de la gravité $\left(\mathrm{m}_{\mathrm{s}} \mathrm{s}^{-2}\right)$ \\
$A r$ & nombre d'Archimède \\
$t$ & temps (s) \\
$x, y$ et $z$ & coordonnées du centre de gravité du corps $(\mathrm{m})$ \\
$u z$ & vitesse d'ascension moyenne du corps $\left(\mathrm{m} \cdot \mathrm{s}^{-1}\right)$ \\
$R e$ & nombre de Reynolds \\
$R e_{\mathrm{ct}}$ & nombre de Reynolds critique d'instabilité du mouvement du corps \\
$R e_{\mathrm{cs}}$ & nombre de Reynolds critique d'instabilité du sillage \\
$\omega$ & pulsation caractéristique des oscillations $\left(\mathrm{rad} . \mathrm{s}^{-1}\right)$ \\
$S t$ & nombre de Strouhal \\
\hline
\end{tabular}

et de rotation $[8,9]$. La forme du corps est donc un paramètre crucial car elle influence les forces d'inertie propre et ajoutée ainsi que les forces de traînée et de portance.

Aussi avons nous concentré notre étude sur le rôle joué par le rapport de forme $d / h$ (diamètre sur épaisseur) dans la cinématique du corps. Pour ce faire, nous avons utilisé des cylindres de rapports de forme variés $(1,5<d / h<$ 10). Pour chaque rapport de forme, nous avons étudié différents nombres d'Archimède :

$$
A r=\sqrt{\frac{\Delta \rho}{\rho} g r_{\mathrm{eq}}} \frac{r_{\mathrm{eq}}}{\nu} \quad \text { avec } \quad \frac{4}{3} \pi r_{\mathrm{eq}}^{3}=\frac{\pi d^{2}}{4} h
$$

Quant au rapport des densités $\left(\rho_{\mathrm{s}} / \rho_{\mathrm{f}}\right)$, il a été fixé pour tous les cas à une valeur proche de l'unité.

\section{Démarche expérimentale}

\subsection{Le dispositif expérimental}

La veine d'étude est constituée d'un canal vertical (1,60 m de haut, section carrée de $40 \mathrm{~cm}$ de côté) fixé sur une structure supportant un chariot mobile verticalement. Le chariot transporte deux caméras perpendiculaires et deux éclairages leur faisant face. Sa position verticale est mesurée à l'aide d'une règle magnétique de haute précision. Deux ordinateurs font l'acquisition, l'un des images prises par les caméras et l'autre de la position verticale du chariot. En bas du canal est disposé un système de lâcher des corps qui est constitué de deux trappes coulissantes commandées de l'extérieur par une molette.

Les cylindres utilisés ont été usinés au laboratoire à partir de barres d'un nylon dont la densité est proche de celle de l'eau $\left(\rho_{\mathrm{s}} \approx 1,020\right.$ g.cm $\left.{ }^{-3}\right)$. Ils ont des diamètres compris entre 5 et $25 \mathrm{~mm}$.

La cuve est remplie d'eau salée avec une concentration ajustée de façon à produire une gamme de nombres
d'Archimède entre 40 et 120, comprenant le seuil d'apparition des instabilités. Cela impose une très faible différence de densité entre le corps et le fluide, entre $5 \times 10^{-3}$ g.cm ${ }^{-3}$ et $10^{-2}$ g.cm ${ }^{-3}$.

\subsection{Le traitement des données}

Les images et la position verticale des caméras sont acquises à une fréquence de $10 \mathrm{~Hz}$, ce qui garantit au moins 25 points de mesure par période d'oscillation. Le traitement numérique des images permet la détection du contour du corps, puis la détermination de son centre de gravité et de son orientation. Notez que la coordonnée verticale $z$ du centre de gravité est obtenue en ajoutant la position verticale du chariot à la mesure obtenue par le traitement d'image. On a ainsi accès à l'évolution tridimensionnelle du centre de gravité (Fig. 1a) et de l'inclinaison de l'axe du corps au cours du temps. Nous avons observé à chaque fois un mouvement de dérive horizontal, toujours inférieur à $2 \%$ de la distance parcourue verticalement et qui semblait ne pas être corrélé aux paramètres physiques. Il est vraisemblablement dû aux inévitables mouvements résiduels du liquide dans la cuve. Nous avons donc décidé de l'éliminer, en filtrant les basses fréquences des signaux mesurés. Les oscillations de la position obtenues après ce traitement (Fig. 1b) sont alors à moyenne nulle.

Même ainsi, leurs amplitudes évoluent encore de manière complexe. Cependant, si on regarde la trajectoire de dessus (Fig. 2a) on peut distinguer un mouvement de précession typiquement 50 fois plus lent que les oscillations principales. Il est possible de déterminer à chaque instant les directions principales d'oscillation dans le plan horizontal à l'aide de la transformée de Hilbert [6]. Une fois projetée dans ce repère principal, l'amplitude des oscillations est maintenant constante, après un court transitoire (Fig. 2b). Il est important de signaler que les oscillations de vitesse horizontale ainsi que celles de l'inclinaison 

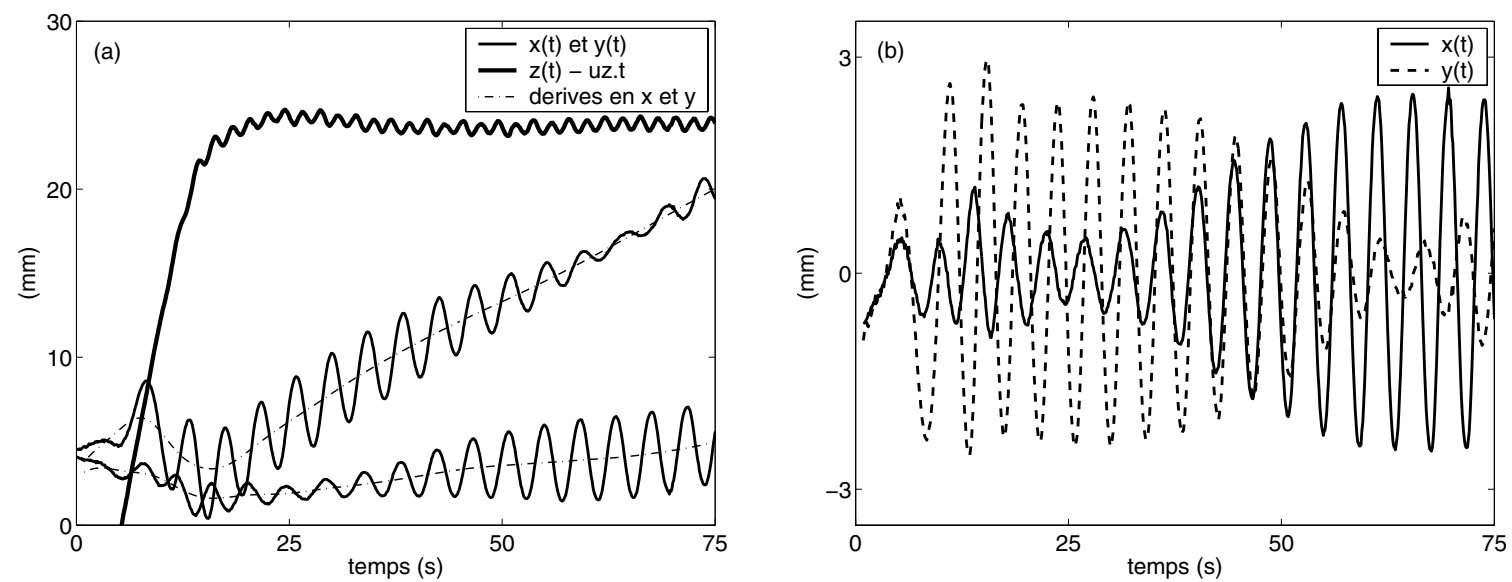

Fig. 1. (a) Positions mesurées; (b) oscillations horizontales sans dérive $(d / h=7, A r=90)$.
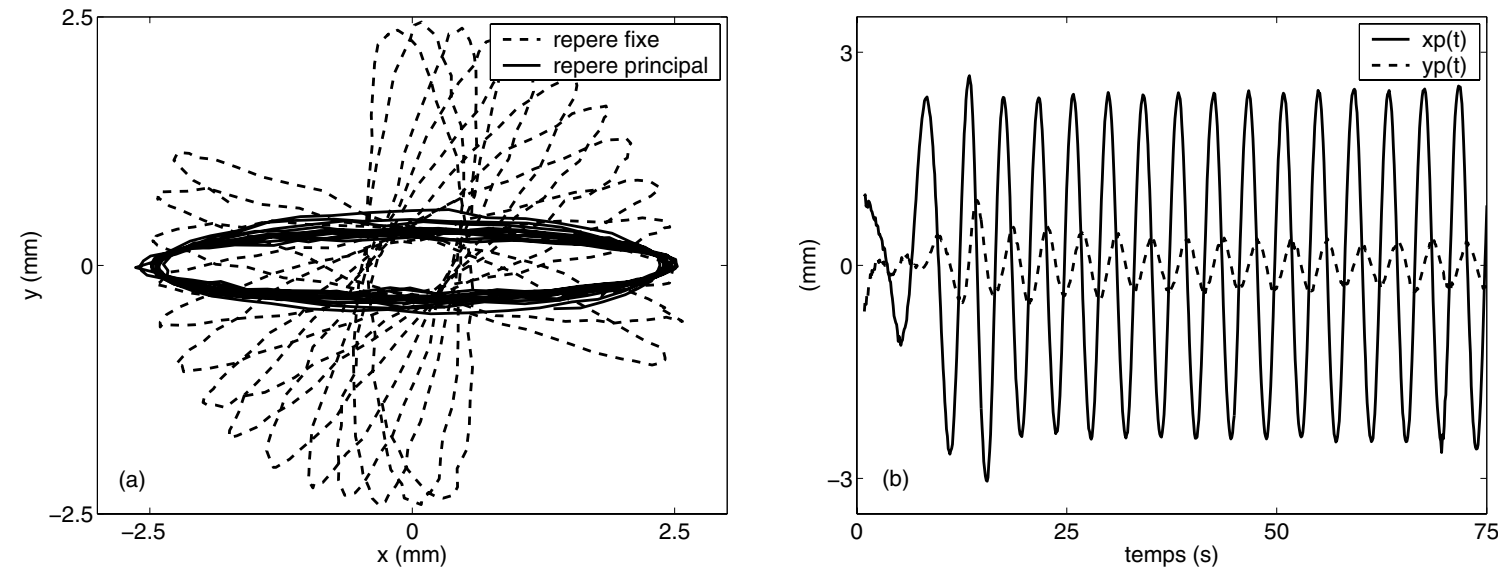

Fig. 2. (a) Trajectoire vue de dessus; (b) oscillations dans le repère principal $(d / h=7, A r=90)$.

sont harmoniques à la même pulsation $\omega$, alors que la vitesse verticale oscille à la fréquence $2 \omega$.

Nous avons appliqué ce traitement à tous les essais, ce qui nous a permis de déterminer les amplitudes et les phases des oscillations des trois composantes de la vitesse du corps et des deux angles définissant l'orientation de son axe.

\section{Résultats expérimentaux}

Nous nous intéressons d'abord à la vitesse moyenne d'ascension adimensionnée sous la forme d'un nombre de Reynolds. La figure 3 a montre que son évolution avec le nombre d'Archimède, pour les différentes valeurs du rapport de forme, se regroupe presque sur une courbe unique. Cela signifie que le coefficient de traînée moyen le long de l'ascension est presque indépendent du nombre de Reynolds et du rapport de forme (Fig. 3b). Le nombre de Reynolds étant mieux adapté à la description hydrodynamique, nous allons désormais l'utiliser, plutôt que le nombre d'Archimède.

À rapport de forme donné, les oscillations apparaissent au-delà d'un certain nombre de Reynolds critique. La figure 4a présente l'évolution de l'amplitude des oscillations de la vitesse transversale ${ }^{1}$ du centre de gravité en fonction du nombre de Reynolds et pour différentes valeurs du rapport de forme. On peut voir que pour un rapport de forme donné il existe un nombre de Reynolds critique $\left(R e_{c t}\right)$ à partir duquel des oscillations auto-entretenues apparaissent.

La figure $4 \mathrm{~b}$ montre comment $R e_{\mathrm{ct}}$ dépend du rapport de forme. On observe deux comportements distincts selon que le rapport de forme est plus grand ou plus petit que 4 . Pour $d / h<4, R e_{\text {ct }}$ diminue lorsque le corps devient de plus en plus mince. Cette évolution est similaire à celle du nombre de Reynolds critique de déclenchement de l'instabilité du sillage $\left(R e_{\mathrm{cs}}\right)$ d'un corps fixe. En effet, $R e_{\mathrm{cs}}$ est plus grand pour une sphère que pour un disque infiniment mince [10]. On remarque aussi que le nombre de Reynolds critique pour un disque fixe est à peu près atteint pour $d / h \approx 4$. Pour $d / h>4$, on retrouve la tendance observée par Willmarth et al. [11] pour des disques très minces $(d / h \gg 1)$ tombant dans un fluide : $R e_{\text {ct }}$ devient une fonction croissante du rapport de forme, s'éloignant du $R e_{\mathrm{cs}}$ du disque mince fixe $\left(R e_{\mathrm{cs}} \simeq 125\right)$. Cependant, même s'il faut un nombre de Reynolds plus grand pour

\footnotetext{
${ }^{1}$ Dans la direction perpendiculaire à la direction de l'axe du
} corps. 

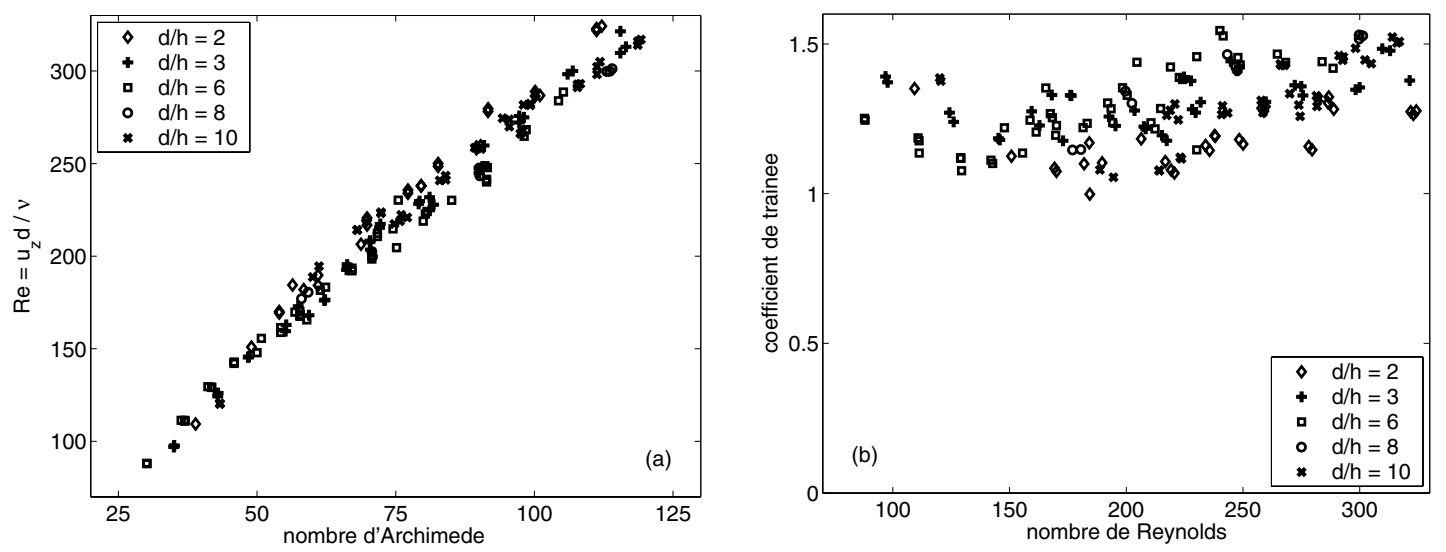

Fig. 3. (a) Nombre de Reynolds; (b) coefficient de traînée moyen.
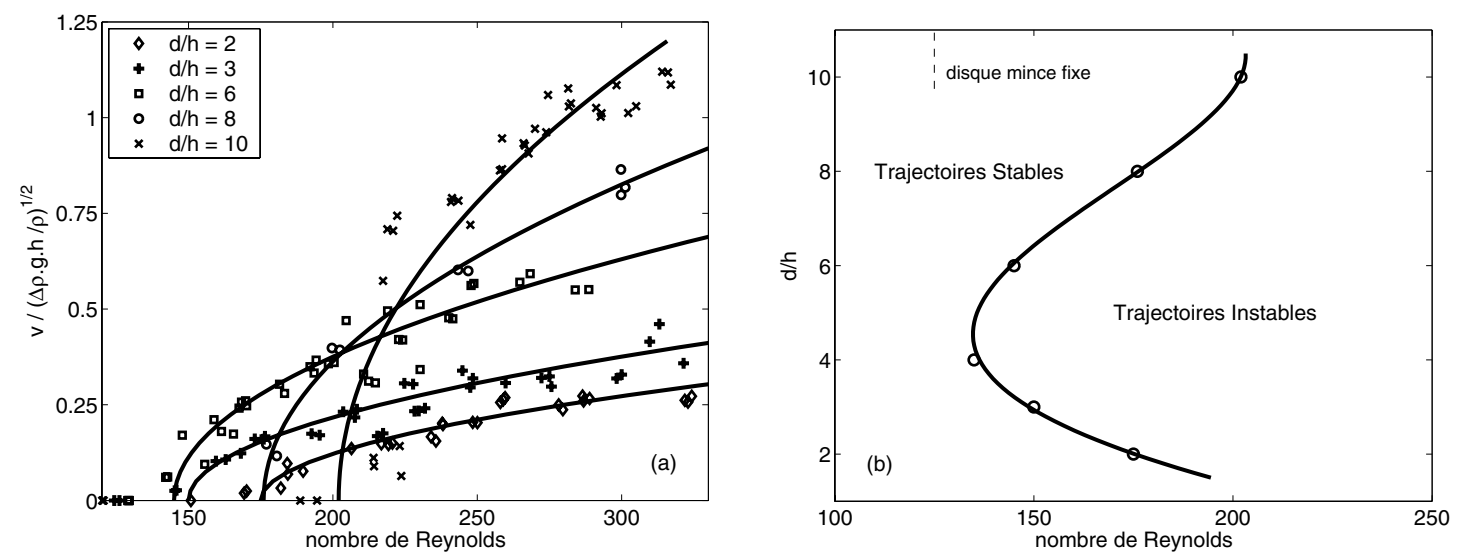

Fig. 4. (a) Amplitudes des oscillations de vitesse tranversale; (b) diagramme de stabilité.

déclencher l'instabilité du mouvement du corps le plus mince, une fois le seuil franchi, les amplitudes de ces oscillations croissent très rapidement, pour atteindre des valeurs beaucoup plus grandes que celles observées pour les corps plus épais.

Nous nous intéressons maintenant à la fréquence du mouvement adimensionnée sous la forme d'un nombre de Strouhal, $S t=\omega d / u_{z}, u_{z}$ étant la vitesse moyenne d'ascension. La figure 5 montre l'évolution du $S t$ en fonction du nombre de Reynolds pour différents rapports de forme.

À rapport de forme donné, le nombre de Strouhal varie peu avec le nombre de Reynolds, mais croît rapidement en fonction du rapport de forme. Les nombres de Strouhal mesurés ici pour des corps mobiles sont proches de ceux correspondant au détachement tourbillonnaire dans le sillage derrière les corps fixes : $S t=0,70$ pour une sphère fixe à $R e_{\mathrm{cs}} \simeq 270$ et $S t=0,79$ pour un disque mince fixe à $R e_{\mathrm{cs}} \simeq 125$.

Même si elles se font à la même fréquence, les oscillations de la vitesse horizontale et de l'orientation du corps ne sont pas, en géneral, en phase. La figure $6 \mathrm{a}$ montre qu'un corps épais a tendance à aligner son axe de révolution avec la vitesse. Au contraire, le corps mince a plutôt tendance à glisser le long de sa trajectoire (Fig. 6b).

La figure 6c montre qu'entre ces deux comportements opposés, ce déphasage évolue continûment d'une

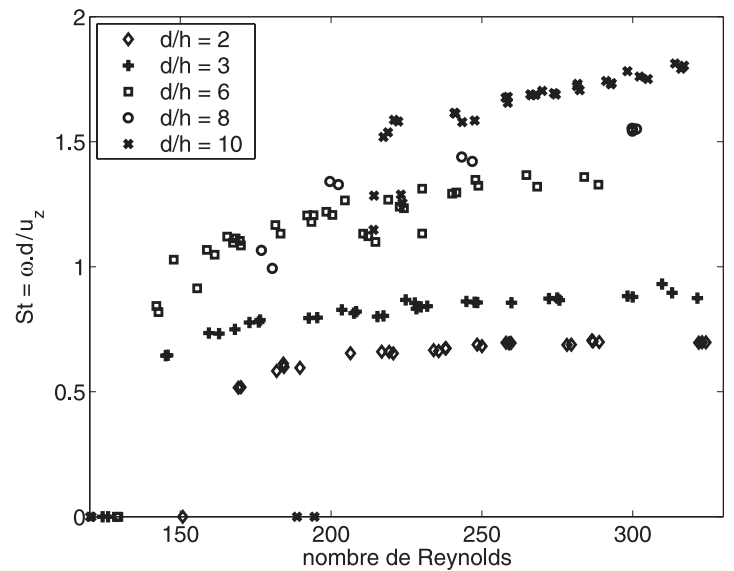

Fig. 5. Nombre de Strouhal fonction du nombre de Reynolds.

valeur légèrement négative jusqu'à une valeur légèrement supérieure à 90 degrés pour $d / h=10$. En revanche, ce déphasage ne dépend presque pas du nombre de Reynolds.

Nous travaillons actuellement sur la dynamique de ces mouvements en essayant de modéliser les forces principales en jeu, afin d'obtenir un système dynamique capable de reproduire le comportement des corps. 

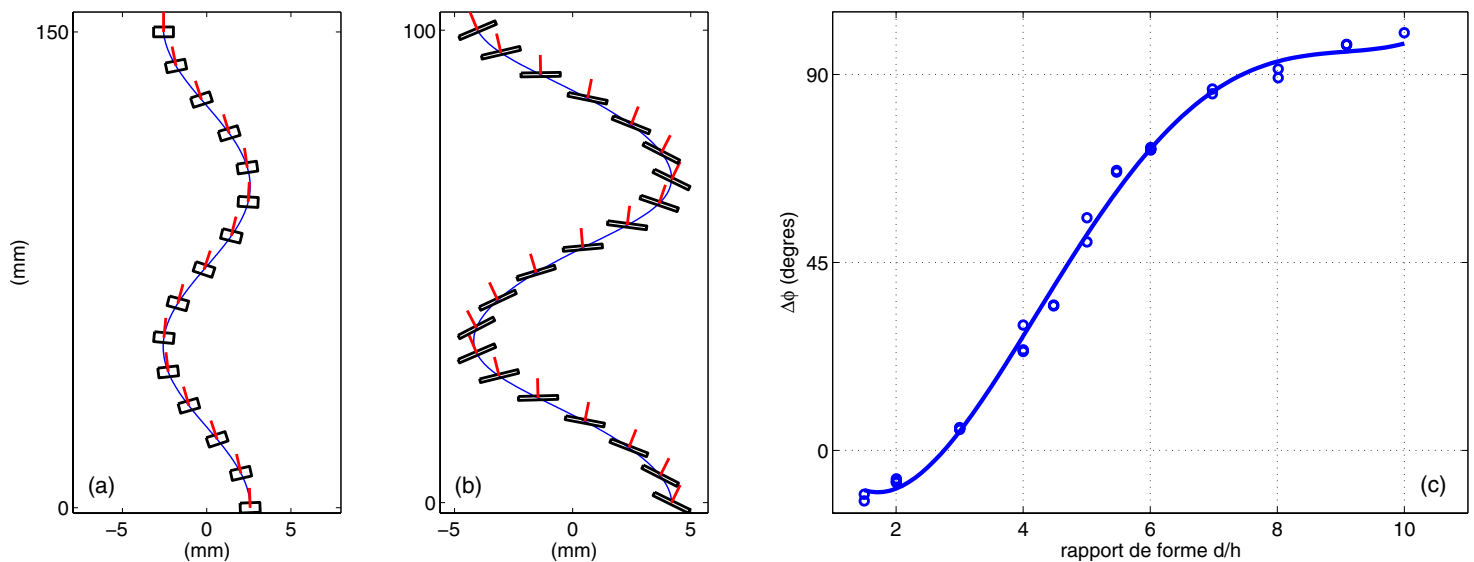

Fig. 6. (a) Mouvement d'un corps épais $(d / h=2)$; (b) mouvement d'un corps mince $(d / h=8)$; (c) évolution du déphasage vitesse/orientation en fonction de $d / h$ pour $R e=250$.

Remerciements. Nous remercions J.-J. Huc et J.-P. Escafit pour la construction du dispositif expériemental, H. Ayroles et $\mathrm{S}$. Cazin pour leur aide concernant la métrologie et C. Trupin pour son soutien technique au quotidien.

\section{Références}

[1] L. De Vinci, Manuscript F, fol. 37r

[2] I. Newton, Philosophiae Naturalis Principia Mathematica, 1687

[3] J.C. Maxwell, The scientific papers of James Clerk Maxwell, Dover, New York, 1890, pp. 115-118

[4] D.G. Karamanev, The study of free rise of buoyant spheres in gas reveals the universal behaviour of free rising rigid spheres in fluid in general, Int. J. Multiphase flow 27 (2001) 1479-1486
[5] M. Jenny, J. Dusek, G. Bouchet, Instabilities and transition of a sphere falling or ascending in a Newtonian fluid, J. Fluid Mech. 508 (2004) 201-239

[6] K. Ellingsen, F. Risso, On the rise of an ellipsoidal bubble in water: oscillatory paths and liquid-induced vorticity, J. Fluid Mech. 440 (2001) 235-268

[7] G. Mougin, J. Magnaudet, Path instability of a rising bubble, Phys. Rev. Lett. 88 (2002) 1-4

[8] H. Lamb, Hydrodynamics, 6th ed. Cambridge University Press, Cambridge, 1932

[9] G. Mougin, J. Magnaudet, The generalized kirchhoff equations and their application to the interaction between a rigid body and an arbitrary time dependent viscow flow, Int. J. Multiphase flow 28 (2002) 1837-1851

[10] R. Natarajan, A. Acrivos, The instability of steady flow past spheres and disks, J. Fluid Mech. 254 (1993) 323-344

[11] W.W. Willmarth, N.E. Hawk, R.L. Harvey, Steady and unsteady motions and wakes of freely falling disks, Phys. Fluids 7 (1964) 197-209 